\title{
Effects of Total Dissolved Gas Supersaturation in Fish of Different Sizes and Species
}

\author{
Shudan Xue, Yuanming Wang *(D), Ruifeng Liang, Kefeng Li and Ran Li \\ State Key Laboratory of Hydraulics and Mountain River Engineering, Sichuan University, \\ Chengdu 610065, China \\ * Correspondence: wangyuanming1991@126.com; Tel.: +86-15108294267
}

Received: 19 May 2019; Accepted: 5 July 2019; Published: 9 July 2019

check for updates

\begin{abstract}
Two endemic fish in the upper Yangtze River, the Rock Carp (Procypris rabaudi) and Prenant's Schizothoracin (Schizothorax prenanti), were used as research objects in this study to assess the effects of total dissolved gas (TDG) supersaturation on fish of varying sizes. Fish were exposed to TDG-supersaturated water at the levels of 145, 140, 135, 130, and 125\%. The results showed that fish swam slowly, responded clumsily, and then exhibited spiral swimming performance after a period of exposure to TDG-supersaturated water. Fish exhibited exophthalmos, body swelling, gill bleeding, and caudal fin bleeding when they died in the TDG-supersaturated water. With the increase in TDG supersaturation, the tolerance capacity of fish to supersaturated TDG significantly reduced. At high supersaturation, the difference in survival time between species was not significant, while fish with smaller sizes showed greater tolerance capacity. At low supersaturation, the tolerance capacity of fish was mainly affected by species, and the influence of size was relatively small. With the decrease in TDG supersaturation, the catalase (CAT) activity first increased and then decreased. Rock Carp displayed significantly less activity than Prenant's Schizothoracin on exposure to TDG-supersaturated water. At high supersaturation levels, the CAT activity of Prenant's Schizothoracin of small size was greater than that of large Prenant's Schizothoracin. In contrast, small Prenant's Schizothoracin showed less CAT activity at low TDG levels than did large individuals.
\end{abstract}

Keywords: total dissolved gas supersaturation; upper Yangtze river; endemic fish; species; size; catalase

\section{Introduction}

Total dissolved gas (TDG) supersaturation is a physical condition in which the pressures of atmospheric gases in a solution exceed the barometric pressure [1], and this condition often occurs downstream of dams during the flood season [2-4] Large amounts of gases are taken into the downstream energy dissipation pool by high-speed turbulent water. The excess dissolved gas cannot be completely released within a short time due to the hydrostatic and hydrodynamic pressure in the energy dissipation pool, thereby resulting in TDG supersaturation in the downstream river water [5-7]. The influence of TDG supersaturation on river ecosystems has become an ecological environmental issue of global concern [8-10]. Endemic fish in the Columbia River of the United States suffered mortality caused by TDG supersaturation when hydropower structures discharged flows during the flood season [11-13]. Similar phenomena were also found downstream of the Danube River [14]. In recent years, a large number of hydropower projects with a high head (i.e., over $200 \mathrm{~m}$ or even as high as $300 \mathrm{~m}$ ) have been planned or built in the upper Yangtze River $[15,16]$. Field observations have found that the TDG supersaturation downstream of these hydropower projects ranged from $116.0 \%$ (Dachaoshan Dam) to $142.5 \%$ (Gongzui Dam) [5], which led to large-scale fish mortality and prompted serious concern in China [17]. Although the restoration of the ecological environment of the 
Yangtze River has been emphasized since 2018 [18], hydropower development in the upper Yangtze River seems to be continuing, and TDG supersaturation will remain the primary issue that needs to be solved in the near future.

Numerous studies have shown that supersaturated TDG always deteriorates the growth, swimming ability, and hatching of fish [19-22], as well as predation capacity [23]. TDG supersaturation even causes fish mortality when the supersaturation exceeds a certain level. The tolerance threshold of fish to TDG supersaturation is a vital reference index for the management of flood discharge in hydropower projects. To protect endemic fish that live downstream from dams, the United States has promulgated a TDG criterion of $110 \%$ in rivers [24]. For endemic fish in China, especially those inhabiting the upper Yangtze River, the existing research has been relatively simple, and has focused on exploring the response of specific fish to TDG supersaturation [25-28]. To date, no studies have systematically assessed the impact of fish species and size on tolerance to TDG supersaturation.

The upper Yangtze River of China has abundant fish resources. The State Council of China approved the Rare and Endemic Fish National Nature Reserve in the upper Yangtze River in April 2000 to protect the rare and endangered fish that inhabit the upper Yangtze River [29,30]. Three endangered species and 65 endemic species were listed as objects of protection. In this paper, two species of different sizes in the upper Yangtze River were selected to explore the effects of species and size on fish tolerance characteristics to TDG supersaturation.

In addition, through a long period of evolution, organisms have formed antioxidant defense systems to prevent damage from toxic substances [31]. As organisms have been subjected to environmental stress, the antioxidant defense system changed to protect the internal tissues of their body from attack [32]. Catalase (CAT) in the antioxidant defense system was selected as an index in this paper and was used to evaluate the response of fish to TDG supersaturation. CAT activities were determined at different TDG supersaturation levels, and the differences shown in the different species and sizes were analyzed. The results of this paper can provide a basis for determining the tolerance threshold of endemic fish in the upper Yangtze River to TDG supersaturation.

\section{Materials and Methods}

\subsection{Experimental Fish}

Rock Carp (Procypris rabaudi) and Prenant's Schizothoracin (Schizothorax prenanti) are the main fish of economic value that inhabit the upper Yangtze River, and belong to benthic species [33]. The population of these species decreased sharply with hydropower construction. They have reached the third and the second levels of urgent protection, respectively [34].

Healthy fish from the Sichuan Fisheries Research Institute of China were used in the experiments. Before the experiment, fish were held in a tank with saturated equilibrium water $(100 \%$ TDG, $7.5-8.0 \mathrm{mg} / \mathrm{L}$ of dissolved oxygen, water temperature of $20-22{ }^{\circ} \mathrm{C}$, and $\mathrm{pH}$ of $7.1-7.8$ ) for one week. Fish were fed earthworms once per day during domestication, and fasted for $24 \mathrm{~h}$ before the experiment to eliminate the effect of food. Fish were divided into four groups to conduct the following experiments. Detailed information about the fish is shown in Table 1.

\subsection{Acute Lethality Experiment}

TDG-supersaturated water was generated by a circulating system that was described in detail by Wang et al. [26]. Experimental fish were held in five plastic tanks $(65 \mathrm{~cm} \times 40 \mathrm{~cm} \times 30 \mathrm{~cm})$ with TDG-supersaturated water. The TDG levels were set as 145, 140, 135, 130, and 125\%. A control level of 100\% TDG was also simultaneously used. Fifteen fish were placed in each tank and TDG supersaturation levels were measured using a Point 4 Tracker (Point Four Systems, Coquitlam, BC, Canada). Fish were immediately removed from tanks once they were identified as dead. The time of death of the individual was recorded, as was the length and weight. An individual was regarded 
as dead when it had no stress response within $5 \mathrm{~min}$ to belly touching by a thin glass rod [35]. Any abnormal behaviors were also recorded during the exposure process.

Table 1. Length and weight of experimental fish. Values are expressed as the mean \pm SE. Letters following the values indicate the results from a post hoc multiple comparison test between groups. Mean values that do not share a common lowercase letter are significantly different Means not sharing a common lowercase letter are significantly different (ANOVA for length: $F=20.70 ; d f=3,289$; $p<0.001$. ANOVA for weight: $F=157.97 ; d f=3,269 ; p<0.001)$.

\begin{tabular}{ccccc}
\hline Group & Experimental Fish & $\boldsymbol{n}$ & $\begin{array}{c}\text { Length } \\
\mathbf{( c m )}\end{array}$ & $\begin{array}{c}\text { Weight } \\
\mathbf{( g )}\end{array}$ \\
\hline small RC & Rock Carp (RC) & 74 & $5.87 \pm 0.09^{\mathrm{d}}$ & $3.57 \pm 0.15^{\mathrm{d}}$ \\
large RC & Rock Carp & 75 & $9.04 \pm 0.13^{\mathrm{a}}$ & $13.53 \pm 0.62^{\mathrm{a}}$ \\
small PS & Prenant's Schizothoracin (PS) & 71 & $6.38 \pm 0.09^{\mathrm{c}}$ & $4.32 \pm 0.16^{\mathrm{c}}$ \\
large PS & Prenant's Schizothoracin & 73 & $8.09 \pm 0.10^{\mathrm{b}}$ & $8.23 \pm 0.29^{\mathrm{b}}$ \\
\hline
\end{tabular}

\subsection{Measurement of CAT Activity}

When a dead fish was removed from the acute lethality experiment, its muscle tissue $(0.15 \mathrm{~g})$ was immediately collected and then lapped with freezing liquid nitrogen. Fresh phosphate buffer solution with a volume of $1.5 \mathrm{~mL}$ was simultaneously added to extract the enzyme solution. The extract enzyme was centrifuged for $3 \mathrm{~min}$ at $4{ }^{\circ} \mathrm{C}$ and $10,000 \mathrm{r} / \mathrm{min}$, and the supernatant was used to determine CAT activity using an ultraviolet spectrophotometer. Three milliliters of reaction mixture $(0.1 \mathrm{~mL}$ of enzyme extract solution, $2.75 \mathrm{~mL}$ of phosphate buffer solution, and $0.15 \mathrm{~mL}$ of $0.6 \%$ hydrogen peroxide solution) were used to determine the absorbance at a wavelength of $240 \mathrm{~nm}$, and the CAT activity was calculated according to the absorbance.

\subsection{Statistical Analysis}

In this paper, the survival rate and average survival time were calculated to reflect the tolerance characteristics of fish to supersaturated TDG. The median lethal time $\left(\mathrm{LT}_{50}\right)$ refers to the exposure time at which the mortality of the test fish reached $50 \%$ under a specific TDG supersaturation level. The $\mathrm{LT}_{50}$ was used to assess the lethality of TDG-supersaturated water to experimental fish. The graphical methods of Miller and Sunter were used to calculate the $\mathrm{LT}_{50}$ at different supersaturation levels [36].

CAT activity was used to evaluate the biological response of fish to TDG-supersaturated water. The activity unit of CAT was defined as the amount of the enzyme that caused a decrease of 0.01 on the absorbance of $\mathrm{H}_{2} \mathrm{O}_{2}$ per min under the wave length of $240 \mathrm{~nm}$, which is expressed as follows:

$$
\text { CATactivity }=\frac{\Delta A \times V_{1}}{0.01 \times V_{2} \times m}
$$

where $\Delta A$ is the absorbance difference value after a 1.5 -min measurement; $V_{1}$ is the total volume of sample liquid, $\mathrm{mL}$; $t$ is the measurement time, $1.5 \mathrm{~min}$; $V_{2}$ is the volume of sample liquid for measurement, $\mathrm{mL}$; and $m$ is the sample mass, $\mathrm{g}$.

The effects of species and size on fish tolerance characteristics were determined using one-way analysis of variance (ANOVA), which was followed by a post hoc multiple comparison test (least significant difference) to determine the difference between the values of the different treatment groups. Tamhane's T2 test was used when there was inhomogeneous variance. The level of significant difference was set to $p<0.05$. 


\section{Results}

\subsection{Acute Lethal Symptoms}

The fish in the TDG-supersaturated water swam normally during the first hour of exposure. Ninety minutes later, the fish swam rapidly and jumped up and down at the TDG supersaturation levels of 145, 140, and 135\%. After $2 \mathrm{~h}$ of exposure, the fish responded clumsily and swam slowly. Spiral swimming performance was found in some fish, and then they gradually lost swimming capacity and remained motionless at the bottom of the tank, finally suffering death. Similar abnormal behavior also existed in the fish at the TDG supersaturation levels of 125 and 130\%, although the behavior started a little later than that in fish at the levels of 145,140 , and $135 \%$. The experimental fish exhibited signs of exophthalmos, body swelling, gill bleeding, and caudal fin bleeding when they died (Figure 1). The scales of some fish were found to be falling off their bodies during the exposure process. No significant difference was found in signs among the four groups.
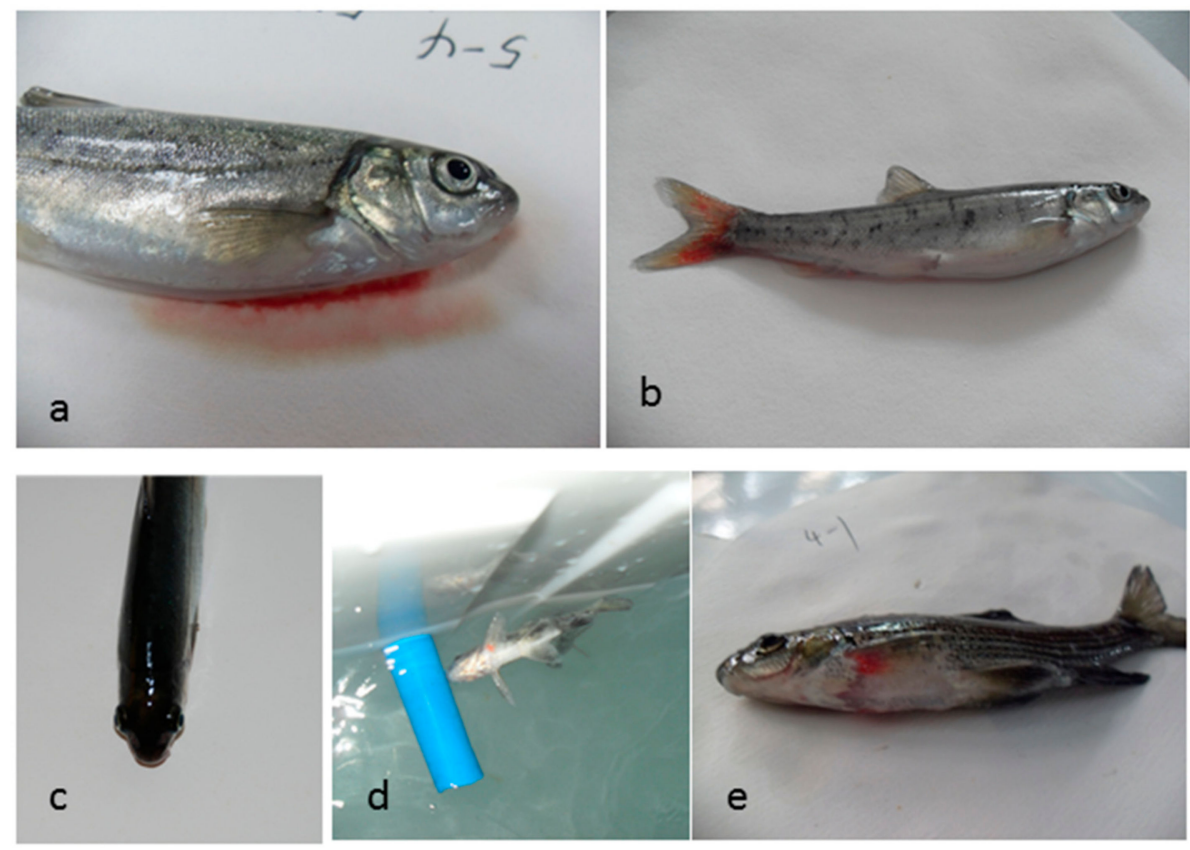

Figure 1. Typical signs of the experimental fish after removal from the total dissolved gas (TDG)-supersaturated water, including (a) gill bleeding, (b) caudal fin bleeding, (c) exophthalmos, (d) spiral swimming, and (e) body swelling.

\subsection{Acute Lethality Experiment}

The survival time of the experimental fish in each group was shorter and more concentrated with increasing TDG supersaturation. For Rock Carp (RC) in the small RC group, the first fish died at between 2.7 and $6.1 \mathrm{~h}$ in the TDG supersaturation levels of 125, 130, 135, 140 and 145\%. As the level of supersaturation increased, the fish died faster. At high supersaturation $(135,140$, and 145\%), $100 \%$ death occurred within 5.3, 3.5, and $3.8 \mathrm{~h}$, respectively, while the time exceeded $14 \mathrm{~h}$ at low supersaturation (125 and 130\%, respectively) (Figure 2a). 

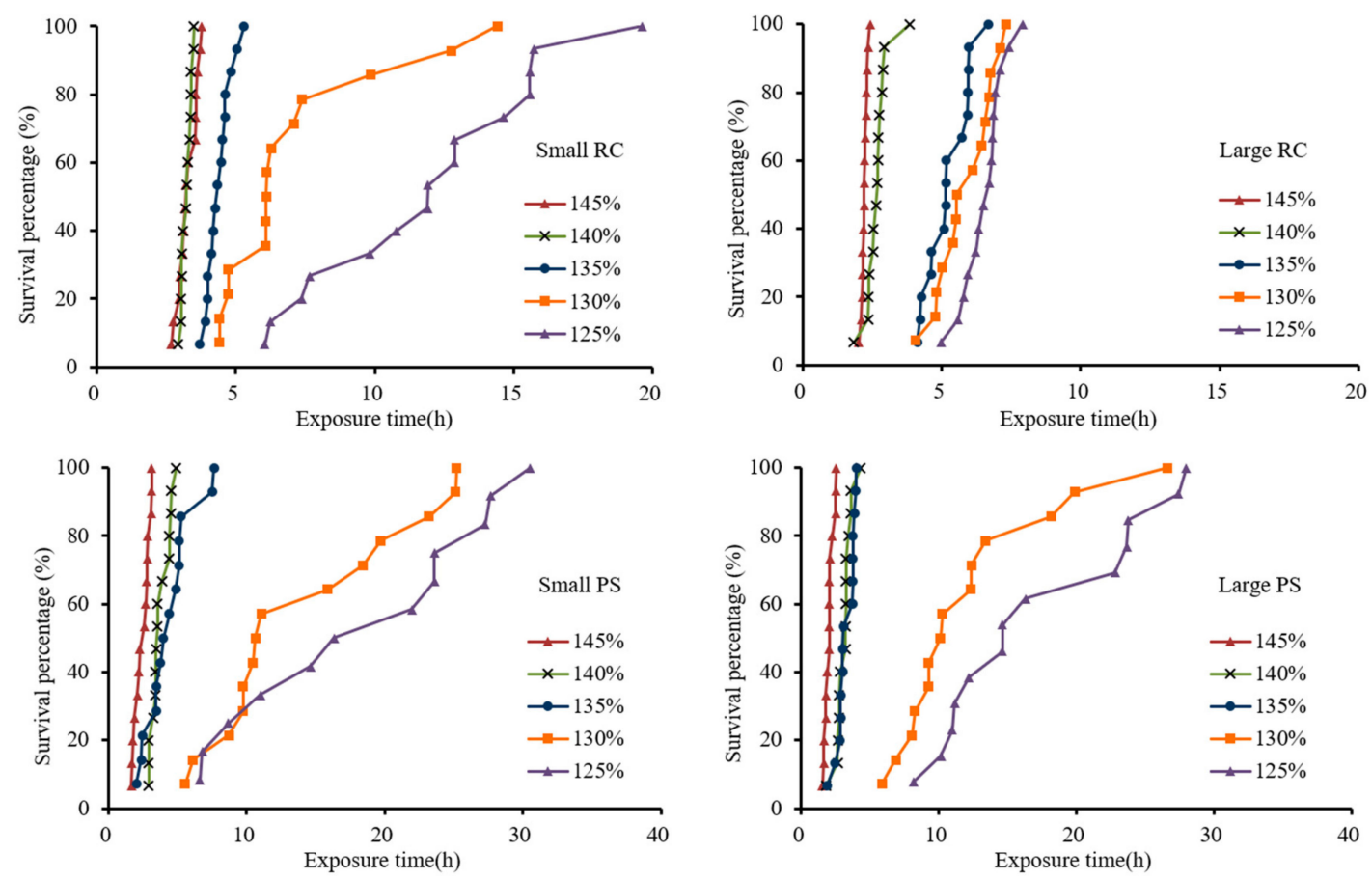

Figure 2. The survival of fish varied with the exposure time at the different TDG supersaturation levels; RC: Rock Carp; PS: Prenant's Schizothoracin.

The survival time of Rock Carp in the large RC group was shorter at the TDG supersaturation levels of 140 and $145 \%$ than in the small RC group. The death times of the first fish in the large RC group were 1.9 and $2.0 \mathrm{~h}$ at the TDG supersaturation levels of 140 and $145 \%$, respectively, and the times to $100 \%$ mortality were 3.9 and $2.4 \mathrm{~h}$, respectively. The experimental fish at the level of $135 \%$ survived longer than did those at 140 and $145 \%$. At the level of $135 \%$, the first fish died at $4.1 \mathrm{~h}$. At the TDG supersaturation level of $135 \%$, there was no significant difference compared with the Rock Carp in the small RC group. Unlike the Rock Carp in the small RC group, the survival of Rock Carp in the large RC group was more concentrated at the levels of 125 and 130\% (Figure 2b).

The survial pattern of Prenant's Schizothoracin was similar to that of Rock Carp. The survival time of Prenant's Schizothoracin was more concentrated at the supersaturation levels of 130, 140, and $145 \%$ than at the levels of 125 and $130 \%$. The mortality rate reached $100 \%$ within $7.6 \mathrm{~h}$ at the levels of 135 to $145 \%$, and Prenant's Schizothoracin (PS) in the small PS group reached 100\% mortality earlier than the fish in the large PS group. At the TDG supersaturation levels of 125 and 130\%, Prenant's Schizothoracin in the small PS group died between 5.6 and $30.5 \mathrm{~h}$, while fish in the large PS group died between 5.9 and $28.7 \mathrm{~h}$, which was significantly longer than the time to mortality for Rock Carp (Figure 2c,d).

The average survival times of Rock Carp in the small RC group were 3.28 and $3.23 \mathrm{~h}$ at the 145 and $140 \%$ supersaturation levels, respectively. No significant difference was found between the two levels in terms of time of death. The average values were 4.4, 7.2, and $11.9 \mathrm{~h}$ at the levels of 135, 130, and $125 \%$, respectively, increasing significantly with the decrease in TDG supersaturation $(F=39.90$; $d f=4,69 ; p<0.001$; Figure 3a). 

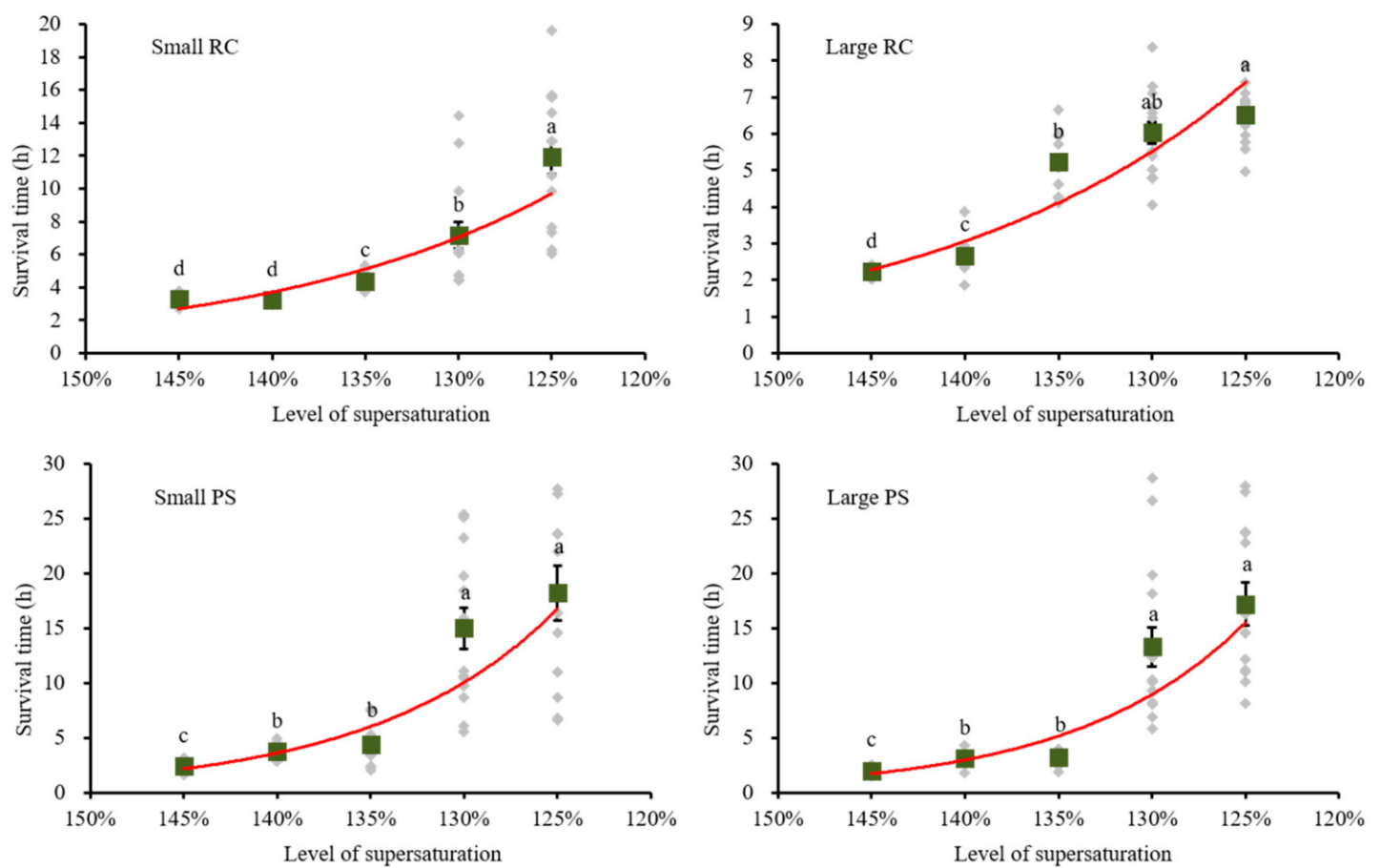

Figure 3. Relationship between survival time and level of supersaturation. Green dots indicate the average survival time. Values are expressed as the mean $\pm \mathrm{SE}, n=12-15$. Gray dots indicate the survival time of the individual. Red lines indicate the best-fit regression models. Letters above the dots indicate the results from a post hoc multiple comparison test between different supersaturation level. Mean values that do not share a common lowercase letter are significantly different $(p<0.05)$.

The average survival times of Rock Carp in the large RC group were 2.2 and $2.7 \mathrm{~h}$ under TDG levels of 145 and $140 \%$, respectively. Similar to the fish in the small RC group, the average survival time of fish in the large RC group increased significantly with the decrease in the TDG supersaturation $(F=108.39 ; d f=4,70 ; p<0.001$; Figure 3b). At the TDG supersaturation levels of 135, 130, and $125 \%$, the average survival times were 5.2, 6.0, and $6.5 \mathrm{~h}$, respectively.

The survival pattern of the small PS group was consistent with that observed in the large PS group. One-way ANOVA indicated that the survival time of fish significantly increased with the decrease in TDG supersaturation (for the small PS group: $F=29.79, d f=4,66, p<0.001$; and for the large PS group: $F=36.39, d f=4,68, p<0.001$; Figure $3 c, d$ ). Post hoc multiple comparisons showed that the survival time at the levels of 140 and $135 \%$ was not significantly different, nor were the survival times at levels of 130 and $125 \%$ significantly different.

At high supersaturation $(145,140$, and 135\%), as shown in Table 2, the difference in the average survival time between species was not significant. Fish with large lengths and weights died more quickly at the TDG supersaturation levels of 145,140 , and $135 \%$, while the difference was significant only at $145 \%$ TDG $(F=35.38 ; d f=3,57 ; p<0.001)$.

Species was the main factor affecting the tolerance capacity of fish at low TDG supersaturation (125 and 130\%) (Table 2). The average survival times of Prenant's Schizothoracin in the small PS group and in the large PS group were greater than those of Rock Carp (ANOVA for 130\%: $F=9.47 ; d f=3,56$; $p<0.001$; ANOVA for 125\%: $F=13.44 ; d f=3,52 ; p<0.001$ ). However, the effect of size on tolerance capacity was not obvious at 125 and 130\% TDG supersaturation. There was no significant difference between Prenant's Schizothoracin in the two groups under the levels of 125 and 130\%. Rock Carp in the two groups also did not show a significant difference at the TDG supersaturation level of $130 \%$. The average survival time of Rock Carp in the small RC group was $5.4 \mathrm{~h}$ longer than that in the large RC group under the TDG supersaturation level of $125 \%$. 
Table 2. Relationship between the average survival time and the TDG supersaturation, which varied with species and size at the different supersaturation levels. Values are expressed the as mean $\pm \mathrm{SE}$, $n=12-15$. Letters following the average survival time indicates the results from a post hoc multiple comparison test between groups at each supersaturation level. Mean values that do not share a common lowercase letter are significantly different $(p<0.05)$.

\begin{tabular}{|c|c|c|c|c|c|}
\hline TDG & Group & $\begin{array}{c}\text { Average } \\
\text { Survival Time }\end{array}$ & df & F & $p$ \\
\hline $145 \%$ & $\begin{array}{l}\text { small RC } \\
\text { large RC } \\
\text { small PS } \\
\text { large PS }\end{array}$ & $\begin{array}{l}3.28 \pm 0.09^{a} \\
2.23 \pm 0.03^{b c} \\
2.44 \pm 0.14^{b} \\
2.02 \pm 0.08^{c}\end{array}$ & 3.57 & 35.38 & $<0.001$ \\
\hline $140 \%$ & $\begin{array}{l}\text { small RC } \\
\text { large RC } \\
\text { small PS } \\
\text { large PS }\end{array}$ & $\begin{array}{l}3.23 \pm 0.05^{\mathrm{a}} \\
2.68 \pm 0.11^{\mathrm{bc}} \\
3.74 \pm 0.17^{\mathrm{b}} \\
3.10 \pm 0.15^{\mathrm{c}}\end{array}$ & 3.60 & 11.71 & $<0.001$ \\
\hline $135 \%$ & $\begin{array}{l}\text { small RC } \\
\text { large RC } \\
\text { small PS } \\
\text { large PS }\end{array}$ & $\begin{array}{c}4.40 \pm 0.11^{\mathrm{a}} \\
5.24 \pm 0.20^{\mathrm{bc}} \\
4.39 \pm 0.46^{\mathrm{b}} \\
3.25 \pm 0.16^{\mathrm{c}}\end{array}$ & 3.55 & 10.10 & $<0.001$ \\
\hline $130 \%$ & $\begin{array}{l}\text { small RC } \\
\text { large RC } \\
\text { small PS } \\
\text { large PS }\end{array}$ & $\begin{array}{l}7.18 \pm 0.82^{a} \\
6.03 \pm 0.30^{b c} \\
15.00 \pm 1.87^{b} \\
13.29 \pm 1.81^{c}\end{array}$ & 3.56 & 9.47 & $<0.001$ \\
\hline $125 \%$ & $\begin{array}{l}\text { small RC } \\
\text { large RC } \\
\text { small PS } \\
\text { large PS }\end{array}$ & $\begin{array}{l}11.91 \pm 1.03^{a} \\
6.53 \pm 0.19^{b c} \\
18.22 \pm 2.49^{b} \\
17.21 \pm 1.93^{c}\end{array}$ & 3.52 & 13.44 & $<0.001$ \\
\hline
\end{tabular}

At a TDG supersaturation level of $145 \%$, the $\mathrm{LT}_{50}$ values of Rock Carp in the small RC and the large RC groups and Prenant's Schizothoracin in the small PS and large PS groups were 2.61, 2.18, 3.40, and $2.19 \mathrm{~h}$, respectively. The $\mathrm{LT}_{50}$ gradually increased with the decrease in TDG supersaturation, and increased to $19.14,16.59,11.14$, and $6.54 \mathrm{~h}$, respectively, at $125 \%$ TDG. There was a negative exponential correlation between the $\mathrm{LT}_{50}$ and TDG supersaturation level $\left(\ln \left(\mathrm{LT}_{50}\right)=12.75-8.21 \mathrm{TDG}, R^{2}=0.787\right)$ (Figure 4).

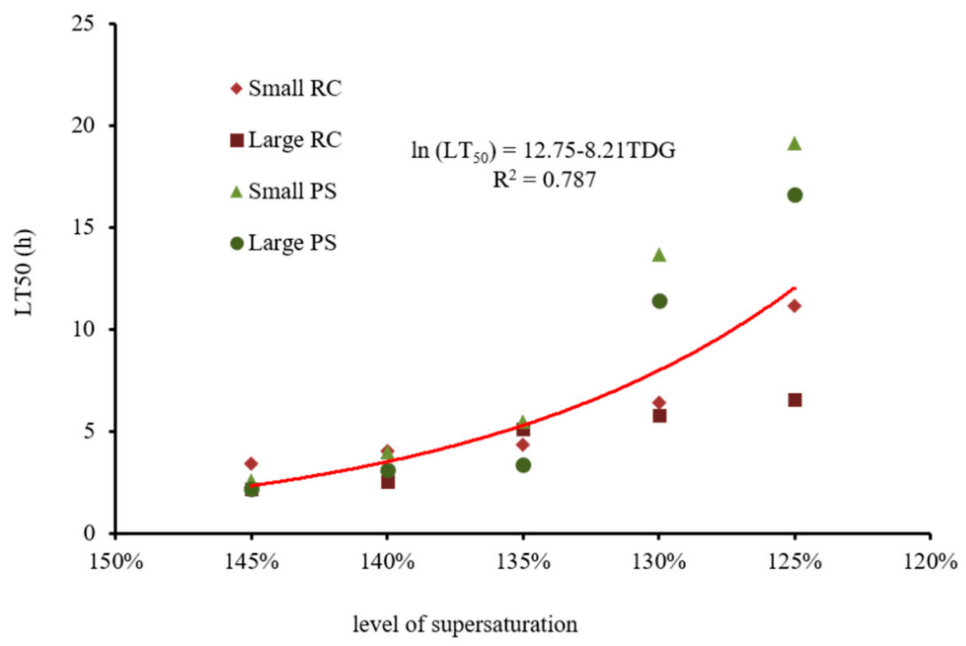

Figure 4. Changes in the median lethal time $\left(\mathrm{LT}_{50}\right)$ of experimental fish with different TDG supersaturation levels. 


\subsection{CAT Activity}

There was no significant difference in CAT activity for Rock Carp in the large RC group at different TDG supersaturation levels $(F=0.97 ; d f=4,36 ; p=0.437)$. Although the CAT activity of Prenant's Schizothoracin in the large PS group changed greatly, with a range of $82.05-116.89 \mathrm{U} / \mathrm{g}$, ANOVA showed that there was no significant difference in CAT activity at different TDG levels $(F=2.41, d f=4$, $62, p=0.059)$. For Prenant's Schizothoracin in the small PS group, the CAT enzyme activity showed no significant difference at high supersaturation levels $(145,140$, and $135 \%)$, and CAT enzyme activity at $130 \%$ was significantly higher than at 145,140 , and $135 \%(F=3.38 ; d f=4,51 ; p=0.016)$.

As shown in Table 3, the results of one-way ANOVA indicated that there were significant differences in CAT activity among fish species at different TDG levels. The average CAT activity of Rock Carp in the large RC group was significantly lower than that of Prenant's Schizothoracin at each TDG level. At high supersaturation levels (145 and 140\%), the average CAT activity of Prenant's Schizothoracin in the small PS group was higher than that of Prenant's Schizothoracin in the large PS group. In contrast, Prenant's Schizothoracin in the small PS group had a lower CAT activity at the TDG levels of 135, 130 and 125\% compared with fish in the large PS group.

Table 3. Catalase (CAT) activities of Rock Carp and Prenant's Schizothoracin at different TDG levels. Values are expressed as the mean \pm SE $(n=5-16)$. Letters following the CAT activities indicates the results from a post hoc multiple comparison test of small PS between different supersaturation levels. Mean values that do not share a common lowercase letter are significantly different $(p<0.05)$.

\begin{tabular}{cccccc}
\hline TDG & Group & CAT Activities & df & F & $p$ \\
\hline \multirow{2}{*}{$145 \%$} & large RC & $34.82 \pm 4.27$ & & & \\
& small PS & $74.58 \pm 7.15^{\mathrm{a}}$ & 2,34 & 8.8 & 0.001 \\
& large PS & $82.05 \pm 8.69$ & & & \\
\hline \multirow{2}{*}{$140 \%$} & large RC & $32.38 \pm 4.70$ & & & \\
& small PS & $85.33 \pm 13.36^{\mathrm{a}}$ & 2,27 & 9.97 & 0.001 \\
& large PS & $102.56 \pm 9.41$ & & & \\
\hline \multirow{2}{*}{$135 \%$} & large RC & $38.52 \pm 4.94$ & & & \\
& small PS & $70.00 \pm 8.09^{\mathrm{a}}$ & 2,31 & 16.34 & 0.001 \\
& large PS & $88.10 \pm 6.18$ & & & \\
\hline \multirow{2}{*}{$130 \%$} & large RC & $47.33 \pm 8.22$ & & & \\
& small PS & $120.00 \pm 14.73^{\mathrm{b}}$ & 2,38 & 8.7 & 0.001 \\
& large PS & $116.89 \pm 11.04$ & & & \\
\hline \multirow{2}{*}{$125 \%$} & large RC & $35.56 \pm 6.36$ & & & \\
& small PS & $124.00 \pm 29.18^{\text {ab }}$ & 2,20 & 6.54 & 0.007 \\
& large PS & $87.22 \pm 10.84$ & & & \\
\hline
\end{tabular}

The average CAT activity at 130\% TDG for all test fish was higher than that of the control group, indicating that the CAT activity was stimulated. In contrast, at the high TDG levels of 135-145\%, most test fish had lower CAT activity relative to control fish, except for Prenant's Schizothoracin in the small PS group at $140 \%$ TDG. This result illustrated that the CAT activity of fish was inhibited at higher supersaturation levels. However, it must be noted that there was no significant difference in the mean value of enzyme activity at each supersaturation level (Table 4). 
Table 4. Comparison of CAT activity between treatment fish and control fish. Bold numbers indicate that the enzymatic activity of this group was higher than that of the control group.

\begin{tabular}{cccc}
\hline TDG & Large RC & Small PS & Large PS \\
\hline $145 \%$ & 34.82 & 74.58 & 82.05 \\
$140 \%$ & 32.38 & 85.33 & 102.56 \\
$135 \%$ & 38.52 & 70.00 & 88.10 \\
$130 \%$ & $\mathbf{4 7 . 3 3}$ & $\mathbf{1 2 0 . 0 0}$ & $\mathbf{1 1 6 . 8 9}$ \\
$125 \%$ & 35.56 & $\mathbf{1 2 4 . 0 0}$ & 87.22 \\
$100 \%$ & 43.34 & 86.67 & 88.79 \\
\hline
\end{tabular}

\section{Discussion}

\subsection{Acute Lethality Experiment}

In this study, at their time of death, the four groups of test fish exhibited exophthalmos, body swelling, gill bleeding, and caudal fin bleeding in supersaturated TDG water, which is in agreement with the research of Wang et al. [26]. During the exposure to TDG supersaturation, the scales of some fish were found to be falling off their bodies. This condition may be caused by furious movement and frequent collisions with the walls of the experimental tanks. No significant difference was found in signs among the four groups, which meant species and size had little effect on the symptoms of gas bubble disease.

At high TDG supersaturation, the test fish in the four groups died rapidly. The death was relatively concentrated, and the TDG supersaturation was the main reason for death. The survival time changed greatly when the supersaturation level was below $135 \%$. We speculate that size and species differences significantly contributed to our results.

Previous studies have shown that different fish species exhibit different tolerance capacities to TDG supersaturation. By comparing the $\mathrm{LT}_{50}$ of five different fish species dwelling in the Columbia River Basin, Beeman et al. concluded that the tolerance of different fish to TDG-supersaturated water was different under the same test conditions [37]. In this paper, species had a greater impact on fish tolerance at 130 and 125\% TDG supersaturation. The tolerance capacity of Rock Carp was significantly weaker than that of Prenant's Schizothoracin. The $\mathrm{LT}_{50}$ of Rock Carp was only 48.6 and $49.5 \%$ of that of Prenant's Schizothoracin at 130 and 125\% TDG supersaturation, respectively.

Size also played an important role in affecting fish tolerance to TDG supersaturation. Robert and Rucker found that the LT50 of the smaller salmon (approximately $38 \mathrm{~mm}$ and $46 \mathrm{~mm}$ ) was approximately 10 times that of the larger salmon (approximately $100 \mathrm{~mm}$ ) [38]. The mortality of salmon with a body length of approximately $100 \mathrm{~mm}$ was approximately $3 / 4$ of that with a body length of $40 \mathrm{~mm}$ [39]. In this paper, both Rock Carp and Prenant's Schizothoracin of larger size had lower LT $_{50}$ than did those of smaller size.

\subsection{CAT Activity}

With the decrease in TDG supersaturation, the CAT activity first increased and then decreased. The maximal value was reached at 130\% TDG, which is in agreement with the law of Chen et al. [31]. At each supersaturation level, the CAT activity of Rock Carp was lower than that of Prenant's Schizothoracin, which indicated that Prenant's Schizothoracin had a stronger ability to decompose excessive $\mathrm{H}_{2} \mathrm{O}_{2}$ than did Rock Carp. Such a result was consistent with the result of $\mathrm{LT}_{50}$, which both indicated that the tolerance capacity of Rock Carp was weaker than that of Rock Carp. For Prenant's Schizothoracin, the CAT activity was higher in fish of larger size at high supersaturation levels (145 and 140\%). In contrast, Prenant's Schizothoracin of larger size had lower activity at TDG levels of 135,130 and $125 \%$.

In this study, the CAT activity of fish was inhibited at high supersaturation. The CAT activity reached a maximum at $130 \%$, and it decreased at a TDG supersaturation level of $125 \%$. It is possible 
that, at high TDG supersaturation, the toxicity of TDG-supersaturated water seriously damaged the bodies of exposed fish. The system of CAT production in fish was damaged, affecting the ability of fish to decompose excess $\mathrm{H}_{2} \mathrm{O}_{2}$. Therefore, the CAT activity of the test fish was lower than that of the control group. At $125 \%$, the external stimulation completely activated the CAT production system. At the TDG supersaturation level of $130 \%$, fish can produce CAT enzymes to resist the toxic effects of TDG supersaturation and decompose excess $\mathrm{H}_{2} \mathrm{O}_{2}$.

\subsection{Suggestions}

This study considered two fish species, and the fish used from both species were juveniles. Considering that they are susceptible to TDG supersaturation, it is important to research their tolerance characteristics to TDG supersaturation. However, such works are not sufficient, and more research should be conducted in the future.

In recent years, an increasing number of high dams have been built and operated in China $[15,16]$. TDG supersaturation is becoming increasingly serious $[8,10,13]$. At present, the amount of research on endemic fish affected by TDG supersaturation in the upper Yangtze River is not rich or sufficient. The evaluation system of the impact of TDG supersaturation on fish needs to be established, and the research work on tolerance to TDG supersaturation needs to be systematic and more profound. The solubility of gases increases with water depth, leading to a decrease in TDG supersaturation. Fish can swim deeper to avoid the threat of TDG supersaturation in natural rivers. When setting the TDG criterion of the upper Yangtze River in the foreseeable future, factors such as species, body shape, life stage, compensation depth, and water temperature should be considered comprehensively $[3,25,26,40,41]$.

\section{Conclusions}

Through the study of the tolerance characteristics of Rock Carp and Prenant's Schizothoracin of different sizes to TDG supersaturation in laboratory, the following conclusions can be drawn:

1. After a period of exposure to TDG-supersaturated water, fish swam slowly, responded clumsily, and then started spiral swimming. Fish exhibited exophthalmos, body swelling, gill bleeding, and caudal fin bleeding when they died in the TDG-supersaturated water. Species and size had little effect on the symptoms of bubble disease.

2. At high TDG levels (135\% and above), the difference in average survival time between different species was not significant, and fish of smaller size showed greater tolerance capacity. At low TDG levels (125 and 130\%), the tolerance capacity of fish to supersaturated TDG was mainly affected by species, and the influence of size was limited.

3. With the decrease in the TDG supersaturation, the CAT activity first increased and then decreased, and reached a maximum value at $130 \%$ TDG supersaturation. The CAT activity was stimulated at levels of $125-130 \%$, and was inhibited at $135 \%$ TDG and above. At different supersaturation levels, the average CAT activity of Rock Carp was significantly lower than that of Prenant's Schizothoracin. At levels of 140 and $145 \%$, the average CAT activity of Prenant's Schizothoracin of small size was greater than that of large Prenant's Schizothoracin. In contrast, small Prenant's Schizothoracin had lower activity than did the large individuals at levels of $125-135 \%$.

Author Contributions: Conceptualization, S.X., Y.W. and K.L.; methodology, S.X.; software, R.L.; formal analysis, S.X. and Y.W.; writing—original draft preparation, S.X.; writing—review and editing, R.L., K.L., and Y.W.

Funding: This research was funded by the National Key Project for Research and Development Plan (2016YFC0401710) and the National Natural Science Foundation of China (51809186).

Conflicts of Interest: The authors declare no conflict of interest.

Ethical Statement: The animal study proposal was approved by the Ethics Committee for Animal Experiments of Sichuan University (2019062101). All experimental procedures were performed in accordance with the regulations for the Administration of Affairs Concerning Experimental Animals approved by the State Council of the People's Republic of China. 


\section{References}

1. Colt, J. Computation of dissolved gas concentrations in water as functions of temperature, salinity and pressure. Am. Fish. Soc. Spec. Publ. 1984, 14, 277-285.

2. Weitkamp, D.E.; Katz, M. A review of dissolved gas supersaturation literature. Trans. Am. Fish. Soc. 1980, 109, 659-702. [CrossRef]

3. Weitkamp, D.E. Total dissolved gas supersaturation biological effects. In Review of Literature 1980-2007; Parametrix Inc.: Bellevue, WA, USA, 2008; pp. 1-65.

4. Qu, L.; Li, R.; Li, J.; Li, K.F.; Deng, Y. Field observation of total dissolved gas supersaturation of high-dams. Sci. China Ser. E Technol. Sci. 2011, 54, 156-162. [CrossRef]

5. Li, R.; Li, J.; Li, K.F.; Deng, Y.; Feng, J.J. Prediction for supersaturated total dissolved gas in high-dam hydropower projects. Sci. China Ser. E Technol. Sci. 2009, 52, 3661-3667. [CrossRef]

6. Politano, M.; Carrica, P.; Weber, L. A multiphase model for the hydrodynamics and total dissolved gas in tailraces. Int. J. Multiph. Flow 2009, 35, 1036-1050. [CrossRef]

7. Wang, Y.; Politano, M.; Weber, L. Spillway jet regime and total dissolved gas prediction with a multiphase flow model. J. Hydraul. Res. 2018, 12, 1-13. [CrossRef]

8. Kamal, R.; Zhu, D.Z.; McArthur, M.; Leake, A. Field Study on the Dissipation of Supersaturated Total Dissolved Gases in a Cascade Reservoir System; World Environmental and Water Resources Congress: West Palm Beach, FL, USA, 2016; pp. 452-460.

9. Witt, A.; Stewart, K.; Hadjerioua, B. Predicting total dissolved gas travel time in hydropower reservoirs. J. Environ. Eng. 2017, 143, 06017011. [CrossRef]

10. Ma, Q.; Li, R.; Feng, J.; Lu, J.; Zhou, Q. Cumulative effects of cascade hydropower stations on total dissolved gas supersaturation. Environ. Sci. Pollut. Res. 2018, 25, 13536-13547. [CrossRef]

11. Arntzen, E.V.; Hand, K.D.; Geist, D.R.; Murray, K.J.; Panther, J.L.; Cullinan, V.I.; Dawley, E.M.; Elston, R.A. Effects of Total Dissolved Gas on Chum Salmon Fry Incubating in the Lower Columbia River; Pacific Northwest National Lab (PNNL): Richland, WA, USA, 2008.

12. Geist, D.R.; Linley, T.J.; Cullinan, V.; Deng, Z.Q. The effects of total dissolved gas on chum salmon fry survival, growth, gas bubble disease, and seawater tolerance. North. Am. J. Fish. Manag. 2013, 33, 200-215. [CrossRef]

13. Witt, A.; Magee, T.; Stewart, K.; Hadjerioua, B.; Neumann, D.; Zagona, E.; Politano, M. Development and implementation of an optimization model for hydropower and Total dissolved gas in the mid-Columbia River System. J. Water Resour. Plan. Manag. 2017, 143, 04017063. [CrossRef]

14. Lechner, A.; Keckeis, H.; Glas, M.; Tritthart, M.; Habersack, H.; Andorfer, L.; Humphries, P. The influence of discharge, current speed, and development on the downstream dispersal of larval nase (Chondrostoma nasus) in the River Danubel. Can. J. Fish. Aquat. Sci. 2018, 75, 247-259. [CrossRef]

15. Zhou, J.P.; Yang, Z.Y.; Chen, G.F. Status and challenges of high dam constructions in China. J. Hydraul. Eng. 2006, 37, 1433-1438.

16. Huang, H.; Yan, Z. Present situation and future prospect of hydropower in China. Renew. Sustain. Energy Rev. 2009, 13, 1652-1656. [CrossRef]

17. CCTV (China Central Television). Shichuan Jinsha Salvage 40 Tons of Dead Fish Poisoning May Exclude Official. Available online: http://news.cntv.cn/2014/07/19/ARTI1405750580177886.shtml.2014. (accessed on 17 July 2014).

18. Cui, L. Hydropower development and ecological environmental protection in Yangtze River basin. J. Hydroelectr. Eng. 2017, 43, 10-12. (In Chinese)

19. Gunnarsli, K.S.; Toften, H.; Mortensen, A. Effects of nitrogen gas supersaturation on growth and survival in larval cod (Gadus morhua L.). Aquaculture 2009, 288, 344-348. [CrossRef]

20. Liu, X.; Li, K.; Du, J.; Li, J.; Li, R. Growth rate, catalase and superoxide dismutase activities in rock carp (Procypris rabaudi Tchang) exposed to supersaturated total dissolved gas. J. Zhejiang Univ. Sci. B 2011, 12, 909-914. [CrossRef] [PubMed]

21. Wang, Y.; Li, Y.; An, R.; Li, K. Effects of total dissolved gas supersaturation on the swimming performance of two endemic fish species in the upper Yangtze River. Sci. Rep. 2018, 8, 10063. [CrossRef] [PubMed]

22. Liang, R.F.; Li, B.; Li, K.F.; Tuo, Y.C. Effect of total dissolved gas supersaturated water on early life of David's Schizothoracin (Schizothorax davidi). J. Zhejiang Univ.Sci. B (Biomed. Biotechnol.) 2013, 14, 632-639. [CrossRef] 
23. Mesa, M.G.; Warren, J.J. Predator avoidance ability of juvenile chinook salmon (Oncorhynchus tshawytscha) subjected to sublethal exposures of gas-supersaturated water. J. Can. Des Sci. Halieut. Et Aquat. 1997, 54, 757-764. [CrossRef]

24. US Army Corps of Engineers. Technical Analysis of TDG Processes; US Army Corps of Engineers-Northwest Division, Environmental Resources and Fish Planning Offices: Kansas City, MO, USA, 2005.

25. Wang, Y.; Liang, R.; Tuo, Y.; Li, K.; Hodges, B. Tolerance and avoidance behavior towards gas supersaturation in Rock Carp Procypris rabaudi with a history of previous exposure. North. Am. J. Aquac. 2015, 77, 478-484. [CrossRef]

26. Wang, Y.; Li, K.; Li, J.; Li, R.; Deng, Y. Tolerance and avoidance characteristics of Prenant's schizothoracin Prenant's Schizothoracin to total dissolved gas supersaturated water. North. Am. J. Fish. Manag. 2015, 35, 827-834. [CrossRef]

27. Cao, L.; Li, K.F.; Liang, R.F.; Chen, S.C.; Jiang, W.; Li, R. The tolerance threshold of Chinese sucker to total dissolved gas supersaturation. Aquac. Res. 2016, 47, 2804-2813. [CrossRef]

28. Yuan, Y.; Wang, Y.M.; Zhou, C.Y.; An, R.D.; Li, K.F. Tolerance of Prenant's schizothoracin Schizothorax prenanti to total dissolved gas supersaturated water at varying temperature. North. Am. J. Aquac. 2018, 80, 107-115. [CrossRef]

29. Fu, C.; Wu, J.; Chen, J.; Wu, Q.; Lei, G. Freshwater fish biodiversity in the Yangtze River basin of China: Patterns, threats and conservation. Biodivers. Conserv. 2003, 12, 1649-1685. [CrossRef]

30. David, D. Requiem for a river: Extinctions, climate change and the last of the Yangtze. Aquat. Conserv. Mar. Freshw. 2010, 20, 127-131.

31. Chen, S.C.; Liu, X.Q.; Jiang, W.; Li, K.F.; Du, J.; Shen, D.Z.; Gong, Q. Effects of total dissolved gas supersaturated water on lethality and catalase activity of Chinese sucker (Myxocyprinus asiaticus Bleeker). J. Zhejiang Univ. Sci. B (Biomed. Biotechnol.) 2012, 13, 791-796. [CrossRef] [PubMed]

32. Liu, X.Q.; Li, K.F.; Jiang, W.; Wu, S. Biochemical responses and survival of rock carp (Procypris rabaudi) to total dissolved gas supersaturated water. Ichthyol. Res. 2015, 62, 171-176. [CrossRef]

33. Ding, R.H. Fishes of the Sichuan; Sichuan Publishing of Science and Technology: Chengdu, China, 1994. (In Chinese)

34. Liu, J. A quantitative analysis on threat and priority of conservation order of the endemic fishes in upper reaches of the Yangtze River. China Environ. Sci. 2004, 24, 395-399.

35. Wilhelms, S.C.; Schneider, M.L. Total dissolved gas in the near-field tailwater of ice Harbor Dam. In Energy and Water: Sustainable Development; ASCE: San Francisco, CA, USA, 1997.

36. Shetty, A.J.; Alwar, M.C. Acute toxicity studies and determination of median lethal dose. Curr. Sci. 2007, 93, 917-920.

37. Beeman, J.W.; Venditti, D.A.; Morris, R.G. Gas bubble disease in resident fish below Grand coulee dam. Final Rep. Res. 2003, 132, 865-876.

38. Robert, R.P.; Rucker, M.K. Effect of Nitrogen Supersaturated Water on Coho and Chinook Salmon. Prog. Fish Cult. 1974, 36, 152-156.

39. Stevens, D.G.; Nebeker, A.V.; Baker, R.J. Avoidance responses of salmon and trout to air-supersaturated water. Trans. Am. Fish. Soc. 1980, 109, 751-754. [CrossRef]

40. Li, X.M.; Fu, S.J.; Li, X.J.; Zheng, H.; Peng, J.L.; Wei, W.; Zhang, Y.G. The effects of meal size on postprandial metabolic response and post-exercise metabolic recovery process in juvenile black carp (Mylopharyngodon piceus). Mar. Freshw. Behav. Physiol. 2018, 51,1-13. [CrossRef]

41. Guzzo, M.M.; Blanchfield, P.J.; Rennie, M.D. Behavioral responses to annual temperature variation alter the dominant energy pathway, growth, and condition of a cold-water predator. Proc. Natl. Acad. Sci. USA 2017, 114, 9912-9917. [CrossRef] [PubMed]

(C) 2019 by the authors. Licensee MDPI, Basel, Switzerland. This article is an open access article distributed under the terms and conditions of the Creative Commons Attribution (CC BY) license (http://creativecommons.org/licenses/by/4.0/). 